Research Paper

\title{
Sleep Deprivation Induces Changes in Immunity in Trichinella spiralis-Infected Rats
}

\author{
Elizabeth G. Ibarra-Coronado시 Javier Velazquéz-Moctezuma², Daniel Diaz ${ }^{3}$, Luis Enrique \\ Becerril-Villanueva ${ }^{4}$, Lenin Pavón ${ }^{4}$ and Jorge Morales-Montor ${ }^{1 凶}$ \\ 1. Departamento de Inmunología, Instituto de Investigaciones Biomédicas, Universidad Nacional Autónoma de México, AP 70228, México D.F. 04510, México. \\ 2. Area de Neurociencias, Departamento de Biología de la Reproducción, CBS, Universidad Autónoma Metropolitana-Iztapalapa, Mexico, DF, México. \\ 3. Departamento de Biología Celular y Fisiología, Instituto de Investigaciones Biomédicas, Universidad Nacional Autónoma de México, México D.F., México. \\ 4. Departamento de Psicoinmunología, Instituto Nacional de Psiquiatria "Ramón de la Fuente," Mexico City, Mexico.
}

$\triangle$ Corresponding author: Dr. Jorge Morales Montor. Departamento de Inmunología, Instituto de Investigaciones Biomédicas, Universidad Nacional Autónoma de México. AP 70228, México DF 04510, México. E-mail: jmontor66@biomedicas.unam.mx, jmontor66@hotmail.com

(C) 2015 Ivyspring International Publisher. Reproduction is permitted for personal, noncommercial use, provided that the article is in whole, unmodified, and properly cited. See http://ivyspring.com/terms for terms and conditions.

Received: 2015.02.16; Accepted: 2015.05.10; Published: 2015.06.06

\begin{abstract}
Sleep is considered an important predictor of immunity. A lack of sleep may reduce immunity, which increases susceptibility to any type of infection. Moreover, sleep deprivation in humans produces changes in both, the percent of circulating immune cells (T cells and NK cells) and cytokine levels (IL-1, IFNy, TN $\Phi-\alpha \alpha$, IL- 6 and IL-17). The aim of our study was to investigate whether sleep deprivation produces deregulation on immune variables during the immune response generated against the helminth parasite Trichinella spiralis. Because sleep deprivation is stressful per se, we designed another experiments to compared stress alone (consisting in movement restriction and single housing) with sleep deprivation, in both control (uninfected) and experimental (infected) rats. Our results demonstrate that the sleep deprivation and stress have a differential effect in mesenteric lymph nodes (MLN) and spleen. In uninfected rats sleep deprivation alone produces an increase in natural killer cells $(\mathrm{NK}+)$ and $\mathrm{B}$ cells (CD45+), accompanied by a decrease in cytotoxic T cells (CD3+CD8+) in spleen; while, in MLN, produces only an increase in natural killer cells $(\mathrm{NK}+)$. Both, SD and stress, produce an increased percentage of total T cells $(\mathrm{CD} 3+)$ in spleen. In the MLN both are also associated to an increase in cytotoxic $T$ cells $(C D 3+C D 8+)$ and $B$ cells $(C D 45+)$. In the spleens of parasitized rats, cell populations did not change. In spleens of both, sleep-deprived and stressed infected rats, we observed an increase in $B$ cells (CD45+). In infected rats, sleep deprivation alone produced an increase in NK cells (NK+). In mesenteric node cell populations of parasitized rats, we observed a decrease in NK cells and an increase in T helper (CD4+) cells in both SD and stressed rats. Rats that were only subjected to stress showed a decrease in $B$ cells (CD45+). These findings suggest that the immune response generated against infection caused by $T$. spiralis is affected when the sleep pattern is disrupted. These results support the notion that sleep is a fundamental process for an adequate and strong immune response generated against this parasite.
\end{abstract}

Key words: sleep deprivation, immune response, parasite, Trichinella spiralis

\section{Introduction}

Despite that sleep function is still not fully understand, there are some functions that seem to be associated to sleep. One of them is the regulation of the immune system and the immune response [1-3].
There are several explanations for the greater susceptibility to infections after reduced sleep, such as impaired mitogenic proliferation of lymphocytes, decreased HLA-DR expression, low antigen uptake, 
decreased phagocytosis, deficient antibody responses, upregulation of CD14+ cells, and variations in CD4+ and CD8+ $T$ cells, which have been observed with partial and total sleep deprivation [3-7].

Some authors propose that sleep participates in the immunological memory consolidation process, $[8$, 9], in which the information is transferred from a short-term to long-term storage and recall phase and participates in the recruitment of antigen-presenting cells to helper T cells $[10,11]$.

Moreover, there are supporting evidences that circulating immune cells and cytokine levels are subject to sleep regulation because their highest systemic levels are reached during sleep, depending on the cell population and interleukin type [12-14]. Populations of cells, such as neutrophils, monocytes and NK cells, are present at their lowest levels in the blood during sleep, but the opposite behaviour is observed in B cells, cytotoxic $\mathrm{T}$ cells and helper $\mathrm{T}$ cells, which reach their highest levels during sleep [12]. On the other hand, rapid eye movement (REM) sleep deprivation, decreases the percent of certain cell populations, such as neutrophils, monocytes and NK cells [12, 15, 16], while total sleep deprivation causes a significant increase in the levels of cytotoxic and helper T lymphocytes [14]. Sleep modulation of immune cell function is demonstrated when sleep deprivation reduces significantly those cytokines produced by specific cell populations, such as T cells, monocytes and dendritic cells (e.g., IL-2, IL-6, IL-1b, IFNY and TNF- $\alpha$ ) $[12,14]$. Other studies conducted in rats that were selectively deprived of REM sleep for a period of $72 h$, reported an increase in the plasma levels of IL-1a, IL1- $\beta$, IL-6, IL-10, TNF- $\alpha$ and IL-17 [17]. These findings suggest that REM sleep deprivation involves changes in the modulation of the immune system and may increase some inflammatory processes.

Despite the fact that sleep and immunity have been extensively studied, little is known of how sleep modulates the immune response. On this respect, subjects who regularly slept the night after vaccination against hepatitis $\mathrm{A}$ in the morning before, increase in antigen-specific antibody titers after 4 weeks later. This effect was confirmed using repeated inoculations with both hepatitis A and B antigens, and it was shown that the effect of nocturnal sleep is on doubling of the number of circulating antigen-specific Th cells against hepatitis A and B, as well as boosting the production of specific antibodies. [3, 18].

However, nothing is known about the effect of sleep deprivation on the development of the immune response against a parasitic infection; particularly, in those parasites that induces gastrointestinal infections, that affects around $70 \%$ of the world population. Nematodes from the Trichinella genus, such as
Trichinella spiralis, the causal agent of porcine and human trichinellosis, are highly evolved parasites that have developed diverse mechanisms for survival within the host that facilitate colonisation. Trichinella spiralis is an intracellular parasitic nematode that invades mammalian striated muscles and it is responsible for trichinellosis, a zoonosis caused by the consumption of raw or undercooked meat from infected animals (e.g., pork) $[19,20]$. The parasite's life cycle includes an infective local phase and a systemic phase. The infection begins with ingestion of contaminated meat muscle larvae to subsequently colonise the small intestine, where they invade the mucosa anchoring to epithelium cells layer. The larvae mature into adults, and once established, females and males copulate, and the females can produce 500-1500 neonate larvae (immature or larvae L1). Newly hatched larvae are able to cross the intestinal epithelium and enter systemic circulation, which results in systemic infection. These larvae are widely distributed in the tissues through the circulation and are eventually established in muscle fibres. Once in muscle fibres, they form the nursing cell and become infectious [20]. Trichinella spiralis infection, in the acute or local phases, causes an enteropathy characterised by hyperplasia of goblet cells, a significant decrease in mucin expression and inflammatory infiltrates in the intestinal lamina [21]. At this stage of infection, there is an intestinal inflammatory process, in which various types of cell populations, including lymphocytes, mast cells and eosinophils, are recruited to the intestinal lamina and lymphatic nodes [22]. The active inflammation in the intestinal mucosa is a Th2 type immune response, suggesting the importance of Th2 cells in protective immunity against this parasite [21, 22]. The acuteness of the symptoms, and the response generated during the enteric phase, can range from imperceptible, to symptoms such as diarrhoea, prostration and malaise, depending on the parasite load with which the host is infected [23, 24].

Sleep deprivation is a stressful condition, independently of the partial sleep deprivation method used. In different animal preparations, various strategies have been developed to reduce the stress component and isolate the lack of sleep (slow-wave sleep or REM sleep or both of them) [25, 26]. Although modifications have been introduced, aimed to reduce the stress component as some indicators are increased, such as corticotrophin releasing hotmone $(\mathrm{CRH})$, adenocorticotrophic hormone $(\mathrm{ACTH})$, and corticosterone serum levels, adrenal weight of all them indicating a general increase in the activation of the hypothalamic-pituitary-suprarenal axis, There is still controversy about the subject, even more if the immune response is assessed, given the marked effect 
of stress on the immune system, mainly the effects produced by cortisol (corticosterone in rats). To distinguish the effects of sleep deprivation, a set of different controls of deprivation techniques has been used, in which the environmental stimuli that can cause stress remain but without inducing sleep deprivation $[25,27]$.

The assessment of the influence of sleep on the development of the immune response involves not only a study of stress as an inherent component of this condition, but also involves a study of the type of response that develops as the immune system responds against infection with a bacteria, virus or parasites. To our knowledge, no data have been published indicating whether sleep is able to affect intestinal immune responses to gastrointestinal worm infection. Thus, the aim of our study was to analyse the effects of sleep deprivation on duodenal T. spiralis colonisation and the systemic percentage of immune cells involved in its control.

\section{Materials and Methods}

\section{Ethics Statement}

Animal care and experimentation practices at the Instituto de Investigaciones Biomédicas and the Universidad Autónoma Metropolitana (UAM) are constantly evaluated by their respective Animal Care and Use Committees, with adherence to official Mexican regulations (NOM-062-ZOO-1999). Mexican regulations are in strict accordance with the recommendations in the Guide for the Care and Use of Laboratory Animals of the National Institute of Health (NIH) of the USA to ensure compliance with the established international regulations and guidelines. The protocol was approved by the Committee on the Ethics of Animal Experiments of the UAM-Iztapalapa. We anesthetized the animals with $\mathrm{CO}_{2}$ and euthanized them by decapitation. Efforts were made to minimise suffering.

\section{Animals and experimental groups}

Male Wistar rats from different cohorts of our colony (200 g weight) were used. All animal groups were singly housed to avoid the risk of infection between groups. The animal room was at a controlled temperature $\left(22-24^{\circ} \mathrm{C}\right)$ and 12-hour light-dark conditions (lights on at $0700 \mathrm{~h}$ ). The diet consisted of Purina rat chow and sterilised water ad libitum.

The animals were divided into the following groups of 7 animals each: 1) uninfected non-sleep-deprived sleep sufficient (intact control), 2) uninfected non-sleep deprived in large platforms and isolated (stress control), 3) uninfected sleep-deprived (SD), 4) infected sleep-sufficient, 5) infected stressed, and 6) infected SD.

\section{Infection}

Trichinella spiralis (ISS 406) has been maintained in our laboratory by serial passage infections in BALB/c mice or Wistar rats. Infective-stage muscular larvae (ML) were recovered from experimentally infected rats at 30 days post infection. Muscles were digested using a standard pepsin-hydrochloric acid digestion method [28]. The muscles of the hind limbs and diaphragm of rats were minced into small pieces. The minced muscle tissues were digested and mixed on magnetic stir plates at $37^{\circ} \mathrm{C}$ for $3 \mathrm{~h}$ in $0.1 \%$ pepsin in $0.1 \% \mathrm{HCl}$ solution, to retain undigested remains, the samples were sieve into conical tubes. Following another 20 min settling, the sediment containing larvae was washed repeatedly for three additional periods of $20 \mathrm{~min}$ in tap water, until the supernatant was clear as water as described in Kapel and Gamble, 2000 [28]

Larvae were recovered and counted under a stereoscopic microscope $40 \times$ magnification to verify for feasibility (motility and kinking) and the average concentration of the number of larvae per $100 \mu \mathrm{l}$ was obtained and adjusted for the inoculation.

Twenty-four hours after the start of sleep deprivation, the infected groups were parasitized. We inserted a gastric catheter and injected 1500 muscular larvae of Trichinella spiralis suspended in $500 \mu \mathrm{l}$ of PBS1x solution directly into the upper part of the stomach. The sleep deprivation procedure continues for forty-eight hours. 72 hours after the start of sleep deprivation (forty-eight hours post-infection) rats were killed by decapitation and adult worms were recovered from the small intestine. The small intestine was dissected, cut longitudinally, and washed twice in 1X PBS. The intestine was then cut into small pieces and incubated in sterile $1 \mathrm{X}$ PBS for 3 hours at $37^{\circ} \mathrm{C}$. Following incubation, the parasites sedimented parasite were collected, washed in PBS1X and counted under a stereoscopic microscope.

\section{Sleep deprivation}

Sleep deprivation was induced using the island technique, which consists on placing rats in rectangular cages with a small central platform $(4.5 \mathrm{~cm}$ diameter and $5 \mathrm{~cm}$ high) surrounded by water at a level of 2.0 to $2.5 \mathrm{~cm}$ from the bottom of the cage, with the water in the cage changed daily as described in Velazquez-Moctezúma, 2004 [29]. With the occurrence of muscle atonia on REM sleep stage, the animal falls into the water and wake up [25]. In accordance with Machado, et al 2005, this method resulted in total sleep REM abolition during the hold deprivation period and slow wave sleep (SWS) loss across 4 deprivation days $(-31 \%)$ [30]. In order to reduce the stress caused by movement restriction, wetness, and muscle 
fatigue, the water level was decrease as necessary to cause minimum arousal, allowing the rats to move within the cage. We add a group who were in similar conditions of isolation and the stress, but in longer platforms (10 cm diameter) to allow animals to sleep.

The experimental protocol for the infected groups included an initial $24 \mathrm{~h}$ of sleep deprivation or stress, after which the groups were infected with 1500 muscular larvae (ML) of Trichinella spiralis. The stress or deprivation procedure continued for an additional $48 \mathrm{hrs}$.

\section{Corticosterone serum levels}

Trunk blood was obtained by decapitation and it was collected in sterile tubes. Two hours after vials were centrifuged at $2300 \mathrm{rpm}$ at $4^{\circ} \mathrm{C}$ for 20 minutes, and serum was collected and frozen at $-80^{\circ} \mathrm{C}$ for further analysis. Corticosterone concentration was determined using a radioimmunoassay kit (Siemens Medical Solutions Diagnostics, Los Angeles, CA, USA). The Coat-A-Count Rat Corticosterone method is a solid-phase 125I-radioimmunoassay, the detection limit of which is $5.7 \mathrm{ng} / \mathrm{mL}$. The samples $(50 \mu \mathrm{L})$ were assayed in duplicate following the manufacturer's instructions.

\section{Flow Cytometry}

Briefly, cells from the spleen or lymph nodes were purified and stained with the following antibodies: anti-rat-CD3-Alexa 647, anti-rat CD4-PE-Cy5, anti-rat CD8-PE, anti-rat CD45RA-PE and anti-rat NK1.1-APC (Biolegend). Cells were fixed with $500 \mu \mathrm{l}$ of fixation buffer (4\% paraformaldehyde, 1X PBS, $\mathrm{pH}$ 7.4). Samples were analysed by flow cytometry using a FACSCalibur (BD Biosciences), and the data were analysed with $\mathrm{FlowJo}^{\circledR}$ software.

\section{Statistical analysis and data processing}

Results were determined in 2 independent experiments. Dependent variables included the number of parasites, serum corticosterone levels, percent of CD3+, CD4+, CD8+, CD19+, and NK cells in the mesenteric lymph nodes and spleen. The independent variable was sleep at three levels:, deprivation or stress or sleep sufficient and infection at two levels: infected or non-infected. Data from 2 replicates $(n=5$ or 4) of each experimental group were expressed as an average \pm standard error and analysed by means of a two way-ANOVA being a study comprising condition and treatment with Bonferroni as a post hoc test. Differences were considered significant when $p<0.05$. Data were analysed using PRISM GraphPad software.

A multiple correspondence analysis (MCA) was applied to determine the complex pattern of association between the differential effect of REM sleep dep- rivation or stress induction, on the percentage of change (increase, decrease or no change) of the immune cell populations $\left(\mathrm{CD}^{+}, \mathrm{CD}^{+}{ }^{+} \mathrm{CD}^{+}, \mathrm{CD}^{+}{ }^{+} \mathrm{CD} 8^{+}\right.$, $\mathrm{CD} 45^{+}$and $\left.\mathrm{NK} 1.1^{+}\right)$in the spleens or mesenteric lymph nodes (MLN) of non-infected and Trichinella spiralis infected rats.

MCA is a multivariate data analytic technique that provides a simple and exhaustive analysis that allows for a detailed description of the data. This analysis incorporates a bidimensional graphical output that displays clouds of points representing the categorised variables [31]. The pattern of correspondence (strength of association) between the variables is interpreted in terms of the relative position (distance) of their points along the two dimensions: $\operatorname{dim} 1$ and dim 2 (factors of variance) defined by the analysis; hence, the closer of the points are represented in space. Each dimension (dim1 or dim2) can explain the pattern of association, and is represented as percentage.

Analysis was performed using Statistical Analysis Software (SAS 9.0, SAS Institute, Cary, NC, USA), and graphs were constructed with Prism 6.0 (GraphPad, Inc.).

\section{Results}

There is not significative difference in the number of adult worms that were recovered from the small intestine of infected groups. The control infected group shown an average of $1180 \pm 137.12$ muscular larvae, the mean for stressed-infected group was $1301 \pm 145.89$ muscular larvae and for the deprivated-infected group was $1175 \pm 148.13$ muscular larvae. When we analysed the intestine of the non-infected groups, we corroborated that no parasites were found. In the sleep deprived group and the stress group do not have significant changes in serum corticosterone concentration. However we found a significant increase produced by the infection, that is not seen in animals that were sleep deprived or stressed (Fig 1).

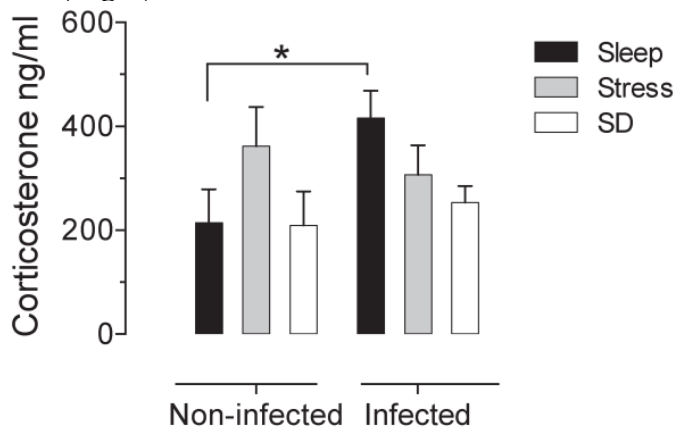

Figure 1. Serum corticosterone concentrations in uninfected (left block) and infected (right blocks) groups in the sleep-sufficient (open bars), stress (shaded bars) and sleep deprivation (SD) (solid bars) conditions. A multiple Student's t test was used to compare statistical significance between the groups. ( $\left.{ }^{*} \mathrm{p}<0.05\right)$. 
Sleep deprivation and stress modify immune cell populations differentially in non-infected rats. Two-way ANOVA analysis and Bonferroni post-test showed the differences statistically significant $(p<$ 0.05). Parasitic infection can enhanced these changes.

T-cells: For the total population of $\mathrm{T}$ cells in the mesenteric lymph nodes (MLN), sleep deprivation caused a decrease of $29 \%$ compared to the stress group $(\mathrm{p}<0.05)$. In spleen, both sleep deprivation and stress caused an increase of 26 and $47 \%(p<0.05)$ in this population (Fig. 2A and 2C). Parasitic infection caused a significant decrease $(27 \%$ in the MLN and $23 \%$ in the spleen) $(\mathrm{p}<0.05)$ in $\mathrm{T}$ cell population, only in stressed animals (Fig. 2B and 2D).

A decrease in the spleen $T$ cell population was also observed as a result of infection in SD and sleep-sufficient groups, $15 \%$ and $6 \%$ respectively, although these values were not statistically significant (Fig 2B and 2D).

The proportions of the $\mathrm{T}$ cell subpopulations were analysed from the total population of $\mathrm{T}$ cells $(\mathrm{CD} 3+)$. The proportions of $\mathrm{T}$ helper cells (CD3+, $\mathrm{CD} 4+)$ and cytotoxic $\mathrm{T}$ cells (CD3+, CD8+) are shown in panels (Fig 3 and 4). For the $\mathrm{T}$ helper cell population $(\mathrm{CD} 4+)$, in infected rats we observed in the MLN an increase of 23 and $31 \%(\mathrm{p}<0.05)$ for the SD and stress conditions, respectively (Fig. $3 \mathrm{~A}$ and $3 \mathrm{~B}$ ). In spleens of infected animals, stress condition caused also an increase of $19 \%$ in CD4+ lymphocytes ( $p$ $<0.05)$.

Furthermore, the CD4+ $\mathrm{T}$ cell subpopulation in the MLN showed a decrease $24 \%(p<0.05)$ in the control infected group, and there was a $10 \%$ increase in $\mathrm{T}$ helper cells in the animals subjected to SD that were subsequently infected, but this result was not statistically significant (Fig. 3B).

In the spleen, the infection caused a $10 \%$ increase in $T$ helper cells $(p<0.05)$ in the stressed animals. However, in this tissue no changes were observed in the control infected group and SD parasitized animals, (Fig 3D).

SD and stress caused the same increase in the cytotoxic $\mathrm{T}$ cell subpopulation (CD8+): a significant increase 28 and $32.5 \%(p<0.05)$ in the MLN of SD and stress groups (Fig. 4A); and only a significant decrease of $16 \%$ in the spleen of the SD group $(p<0.05)$ (Fig. $4 \mathrm{C})$.

In the infected SD animals, there was a significant decrease of $19 \%$ in cytotoxic $\mathrm{T}$ cells in spleen compared to the stressed animals $(\mathrm{p}<0.05)$ (Fig. 4C). In MLN both groups, SD and stressed animals, the infection resulted in a significant decrease in cytotoxic $\mathrm{T}$ cells (32\% for SD and $25 \%$ for stressed animals) (Fig. $4 \mathrm{~B})$, but in the spleen, the infection did not affect the cytotoxic T cell subpopulation (Fig. 4D).
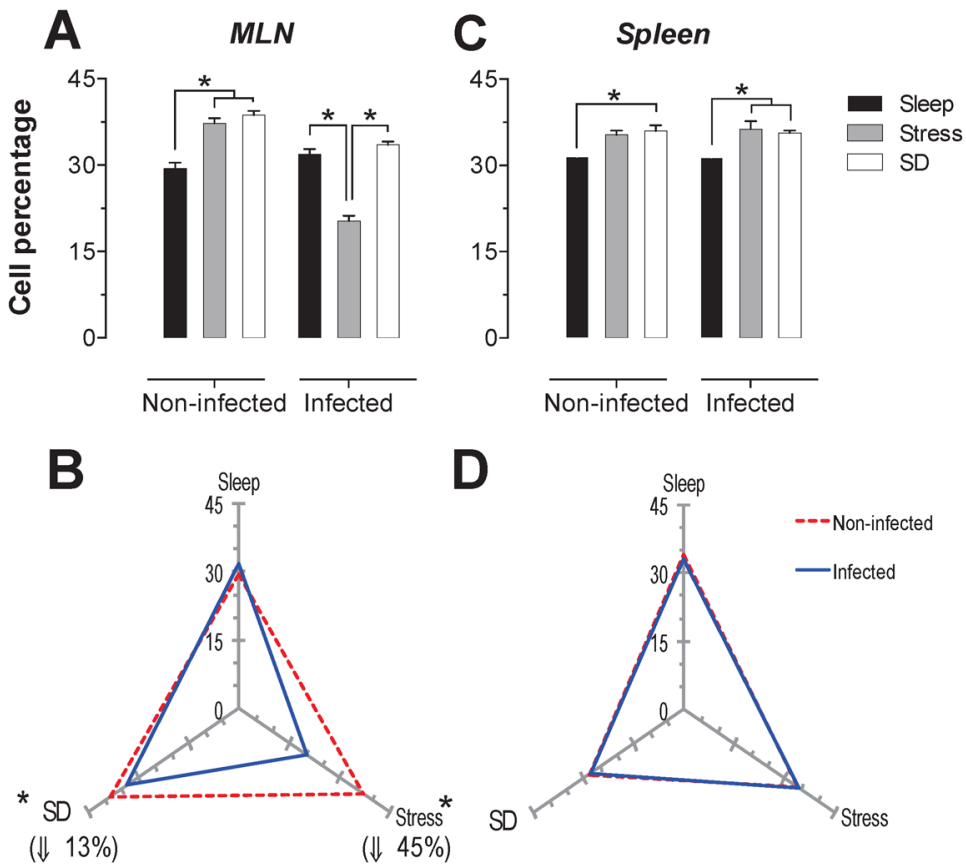

Figure 2. Total T cell (CD3+) percentages in the mesenteric lymph nodes (MLN) and spleen. Sleep-sufficient (open bars), stress (shaded bars) and sleep deprivation (SD) (solid bars) conditions in the MLN (A) and spleen (C) are classified in infected and uninfected groups. Panels $B$ and $D$ show the differential effect of infection in the three study conditions: sleep-sufficient, stress and SD in the MLN (B) and spleen (D). The dashed red line represents the percentage of T cells (CD3+) in uninfected groups; the solid blue line represents the percentage of T cells in the infected groups. ${ }^{*} \mathrm{p}<0.05$ indicates significant differences between the infected vs. uninfected groups. In the uninfected groups, the percentage of T cells decreased in the SD condition compared to the condition of stress in the MLN, whereas in the spleen, SD and stress induced an increase compared to the sleep-sufficient condition. The infection induced a significant decrease only in the stress condition in the MLN, whereas in the spleen, a significant decrease was observed in both the stress condition and the SD and sleep-sufficient conditions. Experimental group were shown as an average \pm standard error and analysed by means of a two way-ANOVA and Bonferroni as a post hoc test. Differences were considered significant when $\mathrm{p}<0.05$. 


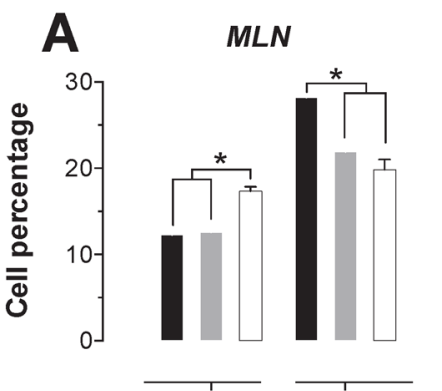

Non-infected Infected

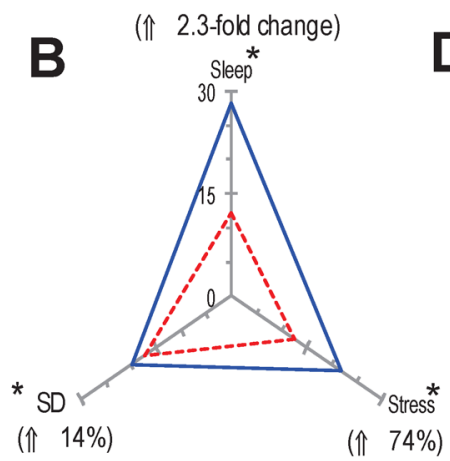

C Spleen

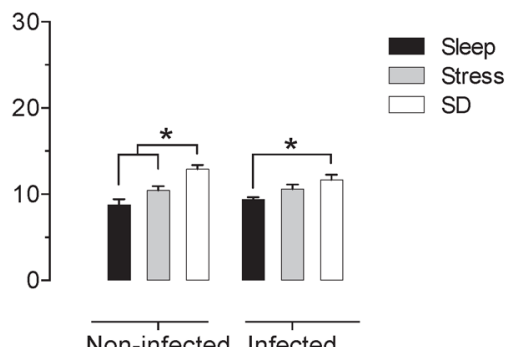

Non-infected Infected

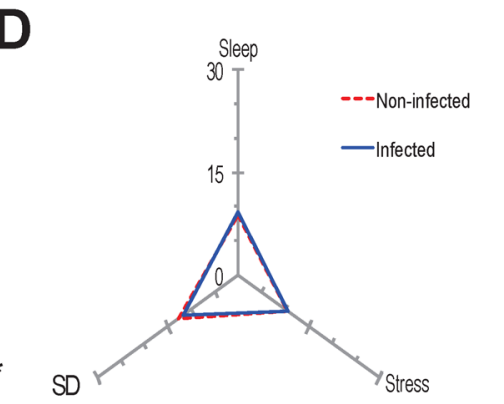

Figure 3. Helper T cells (CD3+CD4+) in the mesenteric lymph nodes (MLN) and spleen. Sleep-sufficient (open bars), stress (shaded bars) and sleep deprivation (SD) (solid bars) conditions in the MLN (A) and spleen (C) were measured in infected and uninfected groups. Panels B and D show the differential effect of infection in the three study conditions: sleep sufficiency, stress and SD in the MLN (B) and spleen (D). The dashed red line represents the percentage of T cells (CD3+) in the uninfected groups; the solid blue line represents the percentage of T cells in the infected groups. ${ }^{*} \mathrm{p}<0.05 \%$; the percentage of change with respect to the uninfected group is shown. In infected groups, the SD and stress conditions increased the percentage of helper T cells compared to the condition of sleep sufficiency in the MLN, whereas in the spleen, only stress induced an increase compared to the sleep-sufficient condition. The infection induced a significant decrease in the sleep-sufficient condition and an increase in the SD condition in the MLN, whereas in the spleen, a significant increase was only observed in the stress condition. Experimental group were shown as an average \pm standard error and analysed by means of a two way-ANOVA and Bonferroni as a post hoc test. Differences were considered significant when $\mathrm{p}<0.05$.

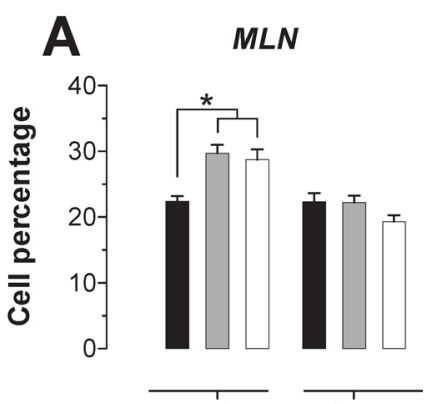

$\overline{\text { Non-infected }} \overline{\text { Infected }}$

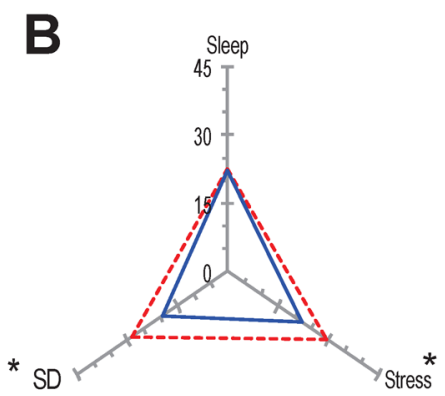

$(\Downarrow 32 \%)$

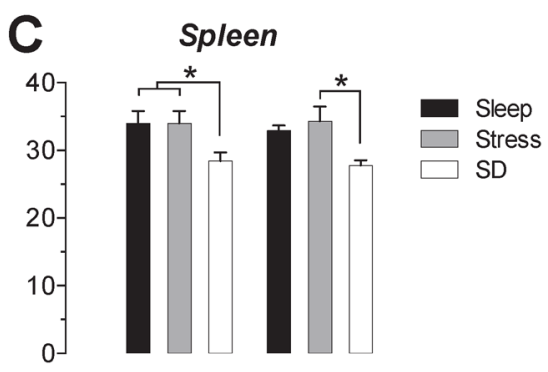

Non-infected Infected

D

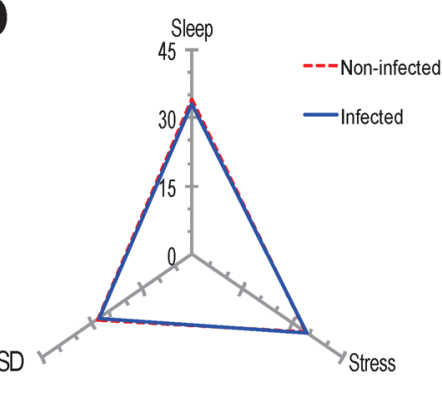

Figure 4. Percentage of cytotoxic T cells $(C D 3+C D 8+)$ in mesenteric lymph nodes (MLN) $(\mathbf{A})$ and spleen $(\mathbf{C})$ for the sleep-sufficient (open bars), stress (shaded bars) and REM sleep deprivation (SD) (solid bars) conditions in infected groups and uninfected groups ${ }^{*} p<0.05 \%$. The differential effect of infection in the three study conditions, sleep-sufficient, stress and SD, is shown in MLN (B) and spleen (D). The dashed red line represents the percentage of T cells (CD3+) in uninfected groups; the solid blue line represents the percentage of T cells in the infected groups. ${ }^{*} \mathrm{p}<0.05 \%$; the percent chance with respect to uninfected is shown. In uninfected groups, the SD and stress conditions increased the percentage of cytotoxic T cells compared to the sleep-sufficiency condition in MLN, whereas in the spleen, SD induced a decrease compared to the sleep-sufficiency and stress conditions; however, in the infected group, the SD decreasing condition was only different during stress. The infection induced a significant decrease in SD and stress conditions and in MLN, whereas in the spleen, the infection appeared to be irrelevant. Experimental group were shown as an average \pm standard error and analysed by means of a two way-ANOVA and Bonferroni as a post hoc test. Differences were considered significant when $\mathrm{p}<0.05$. 


\section{B-cells}

The B cells percentage in MLN presented a significant increase $(p<0.05)$ in the stress and SD conditions of 26 and $31 \%$, in uninfected animals. In the infected rats, only the stressed animals had a significant decrease in CD45+ cells about $36 \%(\mathrm{p}<0-05)$ compared to the sleep-sufficient and SD conditions of infected groups (Fig. 5A). In the spleen of uninfected rats, the SD condition resulted in an increase of $14 \%$ that was significant. The infection in the MLN caused a significant decrease, $13 \%(\mathrm{p}<0.05)$ for the SD condition, while for the stress condition there was a $45 \%$ decrease (Fig. 5B). In the case of spleen, the infection did not cause changes (Fig. 5C), showing that the infection does not appear to alter the modulation exerted by stress and sleep deprivation (Fig. 5D).

\section{NK-cells}

The spleen NK cells showed a significant increase in the SD condition in the infected and uninfected animals about 24 and $46 \%$ respectively (Fig. 6C). In the MLN, in the uninfected groups the SD condition produced a significant increase of $41 \%$ $(p<0.05)$, while in the infected groups, the stress and SD condition caused a significant decrease 24 and 31\% $(p<0.05)$, compared to the sleep sufficient group. The control infected group experienced a significant in-
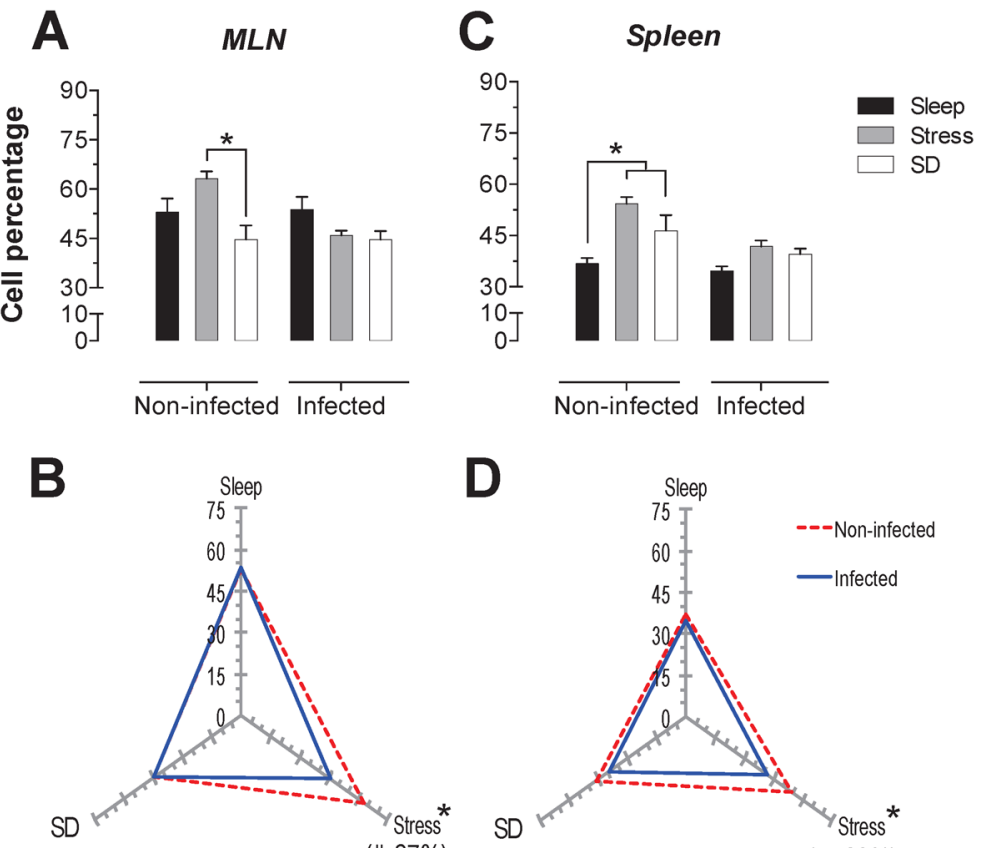

$(\Downarrow 27 \%)$ crease $(p<0.05)$ of 2.3 -fold, while the stress and SD conditions caused an increase $(\mathrm{p}<0.05)$ of $74 \%$ and $14 \%$, respectively (Fig. $6 \mathrm{~B}$ ).

In order to analyse the response of each type of cell population as a function SD and stress conditions in infected and uninfected animals, a correspondence analysis of each tissue, the spleen and MLN, were designed. Multiple correspondence analyses is used to determine the complex pattern of association between a condition and treatment. We applied MCA to determine the differential effect of sleep deprivation or stress (conditions), upon infected or no-infected groups (treatment). This analysis shows the changes associated strongly to a condition detriment. This association is represented by the distance between groups, the strength association between variables is interpreted in terms of the relative positions (distance), of the points of the two dimensions (Dim1 and Dim2). Each dimension can explain the association and is represented as percentage (Fig. 7). For the spleen and MLN, multiple correspondence analysis (MCA) showed the formation of three discrete groups defined by the change (increase, decrease or no change) in the percentage of each cell sub population. However, these groups varied in their composition, suggesting a differential effect provoked by the treatments (SD or stress induction).

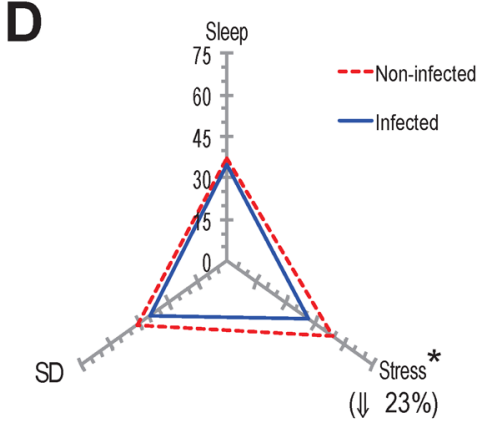

Figure 5. Percentage of B cells (CD 19+) in the mesenteric lymph nodes (MLN) (A) and spleen (C) for the sleep-sufficient (open bars), stress (shaded bars) and sleep deprivation (SD) (solid bars) conditions in infected groups and uninfected groups * $<<0.05 \%$. In panels $B$ and $D$, the differential effect of infection in the three study conditions, sleep-sufficient, stress and SD, in MLN (B) and spleen (D) is shown. The dashed red line represents the percentage of T cells (CD3+) in the uninfected groups; the solid blue line represents the percentage of $T$ cells in the infected groups. ${ }^{*} p<0.05 \%$, the percentage of change with respect to the uninfected group is shown. In the uninfected groups, the SD and stress conditions increased the percentage of B cells compared to the sleep-sufficient condition in the MLN, whereas in the spleen, REMSD induced a decrease compared only to the sleep-sufficient condition. When the animals were infected, in the MLN, only the stress condition produced a significant decrease, but in the spleen, both conditions, REMSD and stress, induced a significant increase. The infection induced a significant decrease in the SD and stress conditions and in the MLN, whereas in the spleen the infection appeared to be irrelevant. Experimental group were shown as an average \pm standard error and analysed by means of a two way-ANOVA and Bonferroni as a post hoc test. Differences were considered significant when $\mathrm{p}<0.05$. 

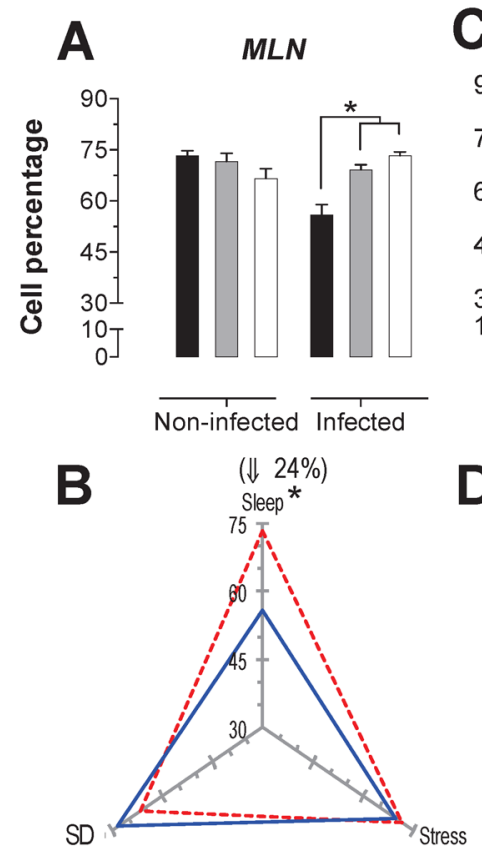

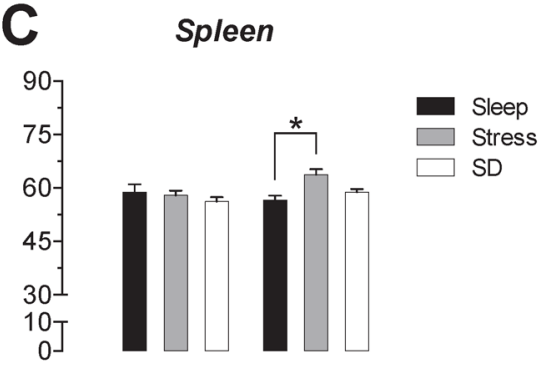

$\overline{\text { Non-infected }} \overline{\text { Infected }}$

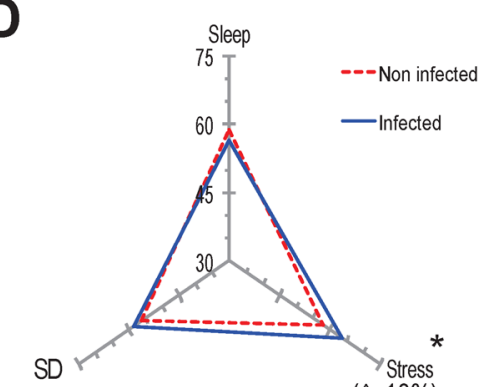

$(\Uparrow 10 \%)$

Figure 6. Percentage of natural killer cells (NK1.1+) in the mesenteric lymph nodes (MLN) (A) and spleen (C) for the sleep-sufficient (open bars), stress (shaded bars) and REM sleep deprivation (SD) (solid bars) conditions in infected groups and uninfected groups * $<<0.05 \%$. Panels $B$ and $D$ show the differential effect of infection in the three study conditions, sleep sufficiency, stress and SD, in the MLN (B) and spleen (D). The dashed red line represents the percentage of T cells (CD3+) in the uninfected groups; the solid blue line represents the percentage of T cells in the infected groups. ${ }^{*}<<0.05 \%$; the per cent change with respect to the uninfected group is shown. In uninfected groups, the SD and stress conditions increased the percentage of NK cells compared to the condition of sleep-sufficiency and stress in the MLN, whereas in the spleen, SD induced an increase compared to both the sleep-sufficient and stress conditions. When the animals were infected, the SD and stress conditions produced a significant decrease in the MLN, but in the spleen, the SD condition induced a significant increase compared to the sleep-sufficient condition. The infection induced a significant increase in the three conditions, and when the animal had sufficient sleep, the infection produced the greatest difference in the MLN, whereas in the spleen, the infection appeared to be irrelevant.
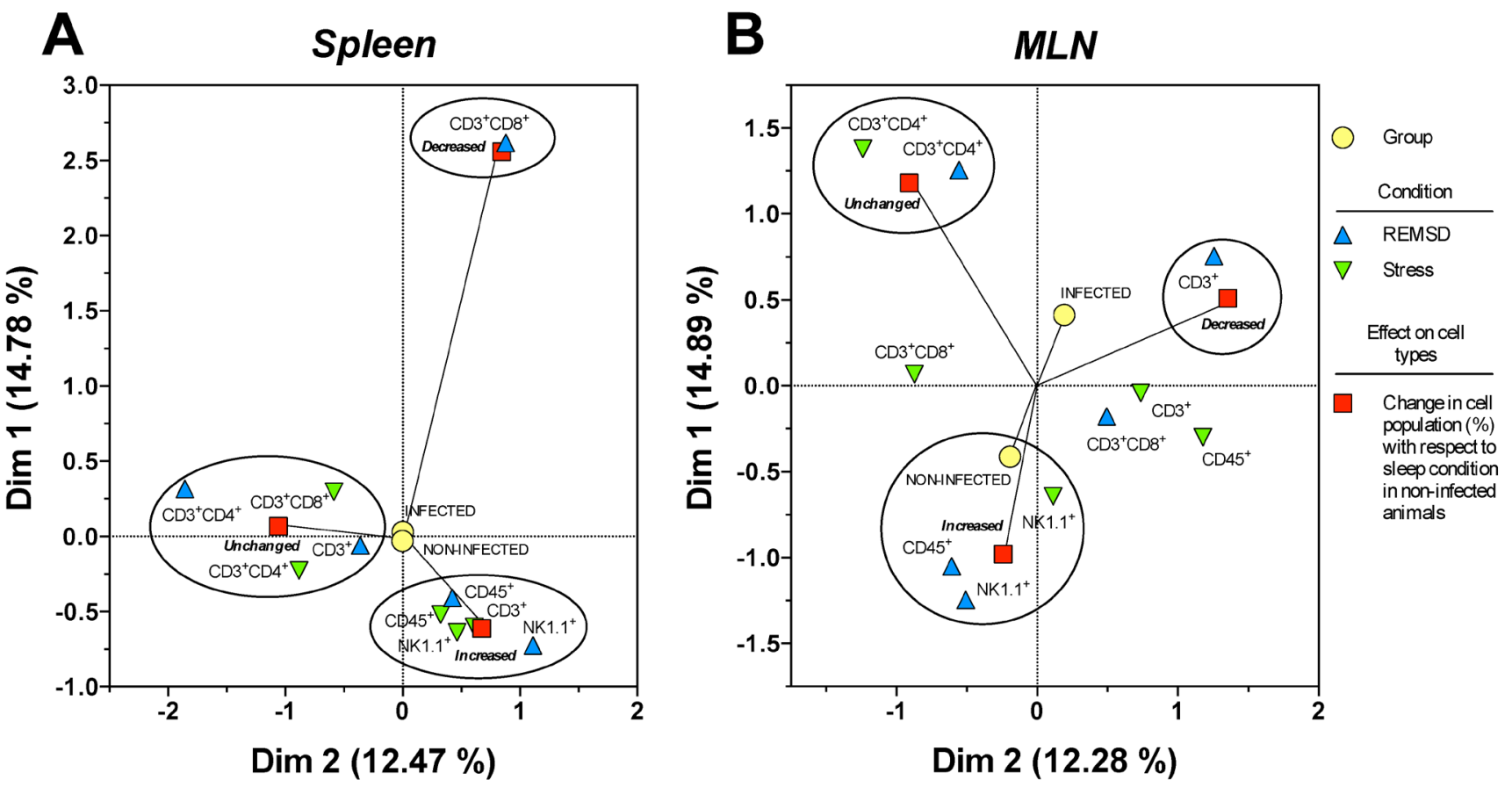

Figure 7. Multiple correspondence analysis of the association pattern between the differential changes in the percentages of circulating immune cell populations and REM sleep (REMSD) deprivation or stress induction in the (A) mesenteric lymph nodes (MLN) and (B) spleen from uninfected rats or rats infected with Trichinella spiralis. The strength of the association between variables can easily be interpreted in relation to the distance separating two adjacent points: the lesser the distance, the greater the association between categories (differential change, cell type, condition and group). The strength association between variables is interpreted in terms of the relative positions (distance), of the points of the two dimensions (Dim1 and Dim2). Each dimension explain the behavior for each cell population analysed, and associates the response to treatment and condition type, this explain is represented as percentage. 
Particularly for the spleen (Fig. 7B), NK1.1 ${ }^{+}$and $\mathrm{CD} 45^{+}$cells were associated to an increase in both conditions, whereas $\mathrm{CD}^{+} \mathrm{CD} 4^{+}$cells were associated with an unchanged response in the SD or stress conditions. Notably, only the $\mathrm{CD}^{+} \mathrm{CD}^{+}$cells corresponded with a decreased response in the sleep deprivation condition. Therefore, the most notable response imposed by sleep deprivation or differentially maintaining or increasing specific circulating immune cell types represented stress induction on the cell populations. Neither the infected nor the uninfected conditions had an association with a particular response; as a consequence, the observed effect on the percentage of the cell populations depended solely on the sleep deprivation or stress conditions for the spleen (Fig. 7B).

MCA for the mesenteric lymphatic nodes showed that unlike the spleen (Fig.7A), the infection with Trichinella spiralis in conjunction with the differential change in the cell populations contributed to the formation of the three groups of responses depicted in Figure 7A. Although the groups were dissimilar in the cells they included, as with the spleen, in the MLN from the SD group, there was an increase in NK1.1 $1^{+}$and $\mathrm{CD} 45^{+}$cells. This increase was associated with the uninfected condition. Consistently, the $\mathrm{CD}^{+}{ }^{+} \mathrm{CD} 4^{+}$cells remained unchanged. There was a group of cells consisting of $\mathrm{CD}^{+}, \mathrm{CD}^{+} \mathrm{CD}^{+}$and $\mathrm{CD} 45^{+}$cells that showed an undefined clear pattern of association between an increase or a decrease in their population percentage, suggesting that the sleep-sufficient condition or the infection produced a varied response in these cells types (Fig. 7A).

\section{Discussion}

Deprivation consists in sleep loss either in a complete lack of sleep during a certain period of time or a optimal sleep time shorter, like to total sleep deprivation, chronic sleep restriction or sleep disruption [32]. Contemporary lifestyle, work-related factors and clinical disorders, insomnia particularly is the most common causes of sleep deprivation; thus the condition affects a considerable number of people [32]. The overall prevalence of insufficient sleep in adults has been estimated at 20\% [33] and furthermore insomnia is the most prevalent sleep disorders and affects severely $10 \%$ of adults worldwide [34]. A chronic reduction in the sleep time or the fragmentation of sleep, leading to the comparable to those of severe acute sleep deprivation; this referring particularly to the cognitive functions, attention and operant memory [35-37].

Although recent research on the effects of sleep deprivation, restriction and disruption on physiological functions in healthy adults, on metabolism, en- docrine function and interesting on immune functions $[6,13,37]$, has been carried out, so far this is the first study exploring the effect of sleep deprivation on the immune response against a gastrointestinal parasite.

In this study, we addressed the influence of sleep deprivation on the behaviour of different cell populations at different levels of organisation, which result in an alteration in the homeostasis of the immune system and therefore lead to an incorrect or poorly mounted immune response. Previous findings have shown that the effectors of the immune response, such as dendritic cells and modulators such as IFN- $\gamma$ and IL-12, which are essential for strengthening the immune response, are under circadian and sleep regulation, and therefore their behaviour can be susceptible to the effects of sleep deprivation [12]. The effects of sleep deprivation on the development of the immune response, and particularly on the acquisition of immunity have been poorly explored. Our study provides evidence of how sleep deprivation modifies the behaviour of different cell populations at different levels of organisation. Our results provide insight into the mechanisms by which sleep deprivation alters the acquisition of immunity, as is the case for the decrease in the generation of antibodies against Hepatitis A vaccine and influenza [18].

A study of the effects of sleep deprivation not only gives us knowledge of the role that sleep may have on different systems in the organism, but it also gives us tools to understand the relationship between different systems, in particular the CNS and immune system, which maintains balance. Understanding the interaction of these systems requires that we include all members of the participating systems. Thus, we cannot study the effect of sleep deprivation on an immune system that remains static, so we demonstrated the effect on physiologically relevant conditions, that is, reacting against an antigenic challenge and interacting with other systems. Despite our extensive work studying sleep deprivation and the immune response, in the context of an antigenic challenge, we must also take into account the influence of infection on other systems. We found that conditions of stress or SD not presented changes, but infection caused an increase in the concentration of corticosterone, we can infer that the infection caused by Trichinella spiralis is more stresfull that the other conditions.

It has been proposed that it is during sleep that different cell subpopulations are recruited into the lymphoid organs, and at the same time sleep is a pro-inflammatory physiological state [11], when we considerate that during night sleep there is an increased production of inflammatory cytokines, such as interleukin-1 (IL-1), tumor necrosis factor alpha 
(TNF- $\alpha$ ), interleukin-12 (IL-12) [13]. Additionally occurs an increased hormone secretion such as growth hormone $(\mathrm{GH})$, prolactin, melatonin and leptin that acts on the immune system by immune cell activation signals support, proliferation, differentiation and the production of the same pro-inflammatory cytokines [11]. Furthermore, it has been shown that sleep deprivation has a differential effect on the different components of the immune system; in particular, it can increase the concentration of NK cells and cytotoxic T cells, as well as pro-inflammatory mediators, such as IL-1 $\beta, T N \Phi-\alpha \alpha$ and IFN $\gamma$ [16]. However, little is known about how sleep deprivation can alter the immune response generated in earlier periods of infection. In this respect, it has been shown that sleep deprivation decreases the generation of antibodies to vaccines against hepatitis A [3], so sleep deprivation indicates the importance of the participation of sleep when an innate immune response and then an adaptive immune response are mounted.

Our findings show that acute sleep deprivation modifies immune cell percentage, and thus, it is logical to propose that immune challenges may alter the normal sleep pattern. The cerebral mechanisms that regulate sleep have reciprocal influences on the endocrine and immune systems. Acute and chronic sleep deprivation modifies the immune response, and conversely, immunological challenges modify the normal sleep pattern. They may be able to modify certain behaviours to facilitate infection and complete their life cycles [38]. However, this field still remains poorly studied, and consequently the function of sleep in the immune response to pathogens beginning to be understood.

A wide variety of research has addressed the importance of sleep for the functioning of the immune system. In 2009, Preston and colleagues analysed the possibility that sleep has evolved across species to allow the organism special protection against parasitic infections. These authors also analysed the correlation between sleep duration and parasitic infection levels as an indicator of the number and type of parasites that are able to infect twelve mammalian species. In this case, a negative correlation was found, with a longer duration of sleep significantly correlating with a lower level of parasitic infection. Thus, the authors conclude that sleep evolved to protect animals from parasitic infections [39].

In this aspect our work addressed the effect of sleep deprivation on the proportion of immune cells in the spleen and MLN at baseline (non-infection) and during a parasitic infection. To this end, the parasite Trichinella spiralis provided a suitable model of infection to evaluate the immune response that is generated in a short period of infection. Our study evalu- ated the effect of sleep deprivation on the behaviour of cells in different compartments, analysing changes in the spleen and MLN, which represent different levels of organisation (or participation) when an immune response is mounted. The changes observed in the MLN give us the first evidence of the importance of sleep on cell recruitment into the peripheral lymph during the innate immune response. Furthermore, the analysis conducted in the spleen cell populations, presents an overview that suggests that sleep deprivation modifies the behaviour of cells at the systemic level in basal conditions as well as during infection.

The controls used showed that stress and SD might differentially affect the behaviour of immune cells. Although SD can be classified as a form of stress, it is mainly characterised by the absence of this essential physiological process, so these stimuli differ in the effects they cause. In this respect, our results showed that in the spleen (reflects systemic effects), the infection status is irrelevant because the infection does not cause a differential effect on the changes observed, that are caused by sleep deprivation or stress, so that the two stimuli produce different responses. Our correspondence analysis revealed that some cell populations respond similarly, but others do not share the same behaviour. Overall, both stress and SD are associated with an increase in the populations of B cells (CD45+) and NK cells (NK1.1+) in the spleen, and stress is also associated with increased total $\mathrm{T}$ cells (CD3+), but in contrast, the SD condition is associated with a decrease in the cytotoxic $\mathrm{T}$ cell subpopulation $(\mathrm{CD} 8+\mathrm{CD} 3+)$. These results are in agreement with those from Velazquez-Moctezuma, 2003, which reported similar changes with different stressors, and therefore, we can postulate that the overall response of the cell subpopulations depends on the immune system nature of the stressor.

When the effects of the infection were analysed, we observed that infection is not able to produce additional changes to those generated by stress or SD in spleen because the infection does not determine the conformation of the groups in MCA analysis (Fig 7A). The increase in B $(\mathrm{CD} 45+)$ cells and NK (NK1.1+) cells relate more to sleep deprivation and stress whist decrease in cytotoxic T cell is clearly related to SD.

In MLN the infection contributes to the formation of groups characterized by the type of response (increase, decrease or no change). In non-infected groups the stress and SD condition are related to increase of NK cells, but the SD also is related to increase of $\mathrm{B}(\mathrm{CD} 45+)$ cells. In infected groups, the SD is strongly related to decreased of total $\mathrm{T}(\mathrm{CD} 3+)$ cells. So deprivation or stress differential effects on cell populations analyzed in presence and absence of infection. 
The response that is mounted against this parasite in the MLN is characterised by increased NK cells (NK1.1+) and decreased T helper cells; we observed that the stress condition and SD both affected the response of both subpopulations, because the increase in NK cells (NK1.1+) and decrease in $\mathrm{T}$ helper $(\mathrm{CD} 4+\mathrm{CD} 3+)$ cells observed during infection was abolished in both conditions. In the case of the spleen, infection don't caused changes in addition to those generated by the SD and stress conditions in the subpopulations studied.

Our work also evidenced that the behavior of cell subpopulations depend on the location of the cells and the type of stimulus; in our case we observed that sleep deprivation modify the behavior of cytotoxic $\mathrm{T}$ cells (CD3+CD8+), B cells (CD3+CD8+)and NK cells $(\mathrm{NK}+)$ in the spleen, while in MLN only population NK cells responded to sleep deprivation, whereas the observed changes in the cytotoxic T cells $(\mathrm{CD} 3+\mathrm{CD} 8+)$ and $B$ cells $(\mathrm{CD} 45+)$ were caused by deprivation as stress.

\section{Conclusion}

There is no difference in parasite loads when rats were sleep deprived or stressed compared to the control group. However, regarding different immune cell subpopulations in spleen or mesenteric lymphatic nodes, there were several changes. The sleep deprivation (SD) in the spleen of non-infected rats produces an increase in natural killer cells $\left(\mathrm{NK}^{+}\right)$and $\mathrm{B}$ cells $\left(\mathrm{CD} 45^{+}\right)$, and a decrease in cytotoxic $\mathrm{T}$ cells $\left(\mathrm{CD}^{+} \mathrm{CD}^{+}\right)$, while in the spleens of infected rats, the sleep deprivation alone produced an increase in NK cells $\left(\mathrm{NK}^{+}\right)$, when is compared to the infected control. On the other hand, the sleep deprivation in the MLNs of non-infected rats produces only an increase in natural killer cells $\left(\mathrm{NK}^{+}\right)$, while in infected rats there is no change when compared to infected control. Only the MLNs of rats infected that were subjected to stress showed a increase in $\mathrm{T}$ helper cells $\left(\mathrm{CD}^{+}{ }^{+} \mathrm{CD} 4^{+}\right)$ compared with infected control, Fuerthermore, both $\mathrm{SD}$ and stress, in spleen of non-infected rats, produce an increased percentage of total T cells $\left(\mathrm{CD}^{+}\right)$. In infected rats, we observed an increase in B cells $\left(C D 45^{+}\right)$ versus infected group SD and stress, in MLN both presented an increase in cytotoxic $\mathrm{T}$ cells $\left(\mathrm{CD}^{+}\right)$and $\mathrm{B}$ cells $\left(\mathrm{CD} 45^{+}\right)$. In infected rats, we observed a decrease in $\mathrm{NK}\left(\mathrm{NK}^{+}\right)$cells and an increase in $\mathrm{T}$ helper $\left(\mathrm{CD} 4^{+}\right)$ cells. As for the correlation of all factors interacting together in the spleen, we observed that in infected or non-infected groups, a decrease in citotoxic $\mathrm{T}$ cells $\left(\mathrm{CD}^{+} \mathrm{CD}^{+}\right)$, was strongly associated with sleep deprivation. Both conditions, SD and stress, were associated to increase in NK cells $\left(\mathrm{NK} 1.1^{+}\right)$and B cells $\left(\mathrm{CD}^{+} 5^{+}\right)$, but an additional increase in total $\mathrm{T}$ cells
(CD3+) associated to stress alone.On the other hand, the correlation of all factors in the MLNs, showed that the SD and stress in non-infected groups affect the same cells populations, producing different effects. The response associated to SD was an increase of $\mathrm{B}$ cells $\left(\mathrm{CD} 45^{+}\right)$and NK cells $\left(\mathrm{NK} 1.1^{+}\right)$, while the response to stress is associated to a decrease in NK cells $\left(\mathrm{NK} 1.1^{+}\right)$. In infected grups, both condition had different effects. The SD was associated to increase in total $\mathrm{T}$ cells $\left(\mathrm{CD}^{+}\right)$, but stress has not association to changes in this parameter.

Our study support the theory that sleep plays a critical role in the immune system maintenance and in the development of the immune response, on this case on the early immune response. Sleep deprivation can improves the immune systemic response against $T$. spiralis, since increasing the recruitment of NK cells can be enhanced cell type response against intracellular parasites. However the SD reduces the local immune response causing a decrease in NK cells in MLN. Stress, on another hand does not improves the systemic immune response, and reduces the local immune response causing a disturbance in the recruitment of NK cells and B cells, involved in the development of the acquisition of immunity against parasites.

\section{Acknowledgements}

Financial support: Grant \#176803 was obtained from Programa de Fondos Sectoriales CB-SEP, Consejo Nacional de Ciencia y Tecnología (CONACyT) and Grant IN208715 from Programa de Apoyo a Proyectos de Innovación Tecnológica, Dirección General de Asuntos del Personal Académico, Universidad Nacional Autónoma de México both to J. Morales-Montor. Elizabeth G Ibarra-Coronado is a Ph.D. student in the Programa de Doctorado en Ciencias Biomédicas, Universidad Nacional Autónoma de México, account number 99158819, and had a doctoral scholarship from CONACyT, CVU 288056.

\section{Conflict of interest}

The authors declare no conflicts of interest.

\section{References}

1. Moldofsky H. Sleep and the immune system. Int J Immunopharmacol. 1995; 17: 649-54.

2. Dinges DF, Douglas SD, Hamarman S, et al. Sleep deprivation and human immune function. Adv Neuroimmunol. 1995; 5: 97-110.

3. Lange T, Perras B, Fehm HL, et al. Sleep enhances the human antibody response to hepatitis A vaccination. Psychosom Med. 2003; 65: 831-5.

4. Casey FB, Eisenberg J, Peterson D, et al. Altered antigen uptake and distribution due to exposure to extreme environmental temperatures or sleep deprivation. J Reticuloendothel Soc. 1974; 15: 87-95.

5. Palmblad J, Cantell K, Strander H, et al. Stressor exposure and immunological response in man: interferon-producing capacity and phagocytosis. J Psychosom Res. 1976; 20: 193-9.

6. Spiegel K, Sheridan JF and Van Cauter E. Effect of sleep deprivation on response to immunization. JAMA. 2002; 288: 1471-2. 
7. Dinges DF, Douglas SD, Zaugg L, et al. Leukocytosis and natural killer cell function parallel neurobehavioral fatigue induced by 64 hours of sleep deprivation. J Clin Invest. 1994; 93: 1930-9.

8. Benington JH and Heller HC. Restoration of brain energy metabolism as the function of sleep. Prog Neurobiol. 1995; 45: 347-60.

9. Mackiewicz M, Shockley KR, Romer MA, et al. Macromolecule biosynthesis: a key function of sleep. Physiol Genomics. 2007; 31: 441-57.

10. Dustin ML and Colman DR. Neural and immunological synaptic relations. Science. 2002; 298: 785-9.

11. Besedovsky L, Lange T and Born J. Sleep and immune function. Pflugers Arch. 2012; 463: 121-37.

12. Dimitrov $\mathrm{S}$, Lange $\mathrm{T}$, Nohroudi $\mathrm{K}$, et al. Number and function of circulating human antigen presenting cells regulated by sleep. Sleep. 2007; 30: 401-11.

13. Lange T, Dimitrov $\mathrm{S}$ and Born J. Effects of sleep and circadian rhythm on the human immune system. Ann N Y Acad Sci. 2010; 1193: 48-59.

14. Born J, Lange T, Hansen $\mathrm{K}$, et al. Effects of sleep and circadian rhythm on human circulating immune cells. J Immunol. 1997; 158: 4454-64.

15. Irwin M, Mascovich A, Gillin JC, et al. Partial sleep deprivation reduces natural killer cell activity in humans. Psychosom Med. 1994; 56: 493-8.

16. Zager A, Andersen ML, Ruiz FS, et al. Effects of acute and chronic sleep loss on immune modulation of rats. Am J Physiol Regul Integr Comp Physiol. 2007; 293: R504-9.

17. Yehuda S, Sredni B, Carasso RL, et al. REM sleep deprivation in rats results in inflammation and interleukin-17 elevation. J Interferon Cytokine Res. 2009; 29: 393-8.

18. Lange $\mathrm{T}$, Dimitrov $\mathrm{S}$, Bollinger $\mathrm{T}$, et al. Sleep after vaccination boosts immunological memory. J Immunol. 2011; 187: 283-90.

19. Bruschi F and Murrell KD. New aspects of human trichinellosis: the impact of new Trichinella species. Postgrad Med J. 2002; 78: 15-22.

20. Dupouy-Camet J. Trichinellosis: a worldwide zoonosis. Vet Parasitol. 2000; 93 : 191-200.

21. Khan WI. Physiological changes in the gastrointestinal tract and host protective immunity: learning from the mouse-Trichinella spiralis model. Parasitology. 2008; 135: 671-82.

22. Knight PA, Brown JK and Pemberton AD. Innate immune response mechanisms in the intestinal epithelium: potential roles for mast cells and goblet cells in the expulsion of adult Trichinella spiralis. Parasitology. 2008; 135: 655-70.

23. Kefenie H and Bero G. Trichinosis from wild boar meat in Gojjam, north-west Ethiopia. Trop Geogr Med. 1992; 44: 278-80.

24. Schmitt N, Saville JM, Greenway JA, et al. Sylvatic trichinosis in British Columbia: potential threat to human health from an independent cycle. Public Health Rep. 1978; 93: 189-93.

25. Suchecki D and Tufik S. Social stability attenuates the stress in the modified multiple platform method for paradoxical sleep deprivation in the rat. Physiol Behav. 2000; 68: 309-16.

26. Palma BD, Tiba PA, Machado RB, et al. [Immune outcomes of sleep disorders: the hypothalamic-pituitary-adrenal axis as a modulatory factor]. Rev Bras Psiquiatr. 2007; 29 Suppl 1: S33-8.

27. Fenzl T, Romanowski CP, Flachskamm C, et al. Fully automated sleep deprivation in mice as a tool in sleep research. J Neurosci Methods. 2007; 166: 229-35.

28. Kapel CM and Gamble HR. Infectivity, persistence, and antibody response to domestic and sylvatic Trichinella spp. in experimentally infected pigs. Int J Parasitol. 2000; 30: 215-21.

29. Velazquez-Moctezuma J, Dominguez-Salazar E, Cortes-Barberena E, et al. Differential effects of rapid eye movement sleep deprivation and immobilization stress on blood lymphocyte subsets in rats. Neuroimmunomodulation. 2004; 11: 261-7.

30. Machado RB, Hipolide DC, Benedito-Silva AA, et al. Sleep deprivation induced by the modified multiple platform technique: quantification of sleep loss and recovery. Brain Res. 2004; 1004: 45-51.

31. Costa PB, Granada CE, Ambrosini A, et al. A model to explain plant growth promotion traits: a multivariate analysis of 2,211 bacterial isolates. PLoS One. 2014; 9: e116020.

32. Malik SW and Kaplan J. Sleep deprivation. Prim Care. 2005; 32: 475-90.

33. Hublin C, Kaprio J, Partinen M, et al. Insufficient sleep--a population-based study in adults. Sleep. 2001; 24: 392-400.

34. Leger D and Ogrizek P. Review on sleep and awakening disorders. Rev Med Interne. 2012; 33: 525-31.

35. Van Dongen HP, Maislin G, Mullington JM, et al. The cumulative cost of additional wakefulness: dose-response effects on neurobehavioral functions and sleep physiology from chronic sleep restriction and total sleep deprivation. Sleep. 2003; 26: 117-26.

36. Goel N, Rao H, Durmer JS, et al. Neurocognitive consequences of sleep deprivation. Semin Neurol. 2009; 29: 320-39.

37. Banks $S$ and Dinges DF. Behavioral and physiological consequences of sleep restriction. J Clin Sleep Med. 2007; 3: 519-28.

38. Donovick PJ and Burright RG. The consequences of parasitic infection for the behavior of the mammalian host. Environmental Health Perspectives. 1987; 73: 247-50.

39. Preston BT, Capellini I, McNamara P, et al. Parasite resistance and the adaptive significance of sleep. BMC Evolutionary Biology. 2009; 9: 7. 